\title{
Paraneoplastic syndromes in digestive tumors; a review
}

\author{
TEODORA DRAGHICI ${ }^{1}$, LUCIAN NEGREANU ${ }^{2,3}$, OVIDIU G. BRATU ${ }^{2,4}$, ANCA PANTEA \\ STOIAN $^{2,5}$, BOGDAN SOCEA ${ }^{2,6}$, TIBERIU PAUL NEAGU ${ }^{2,7}$, ANA MARIA ALEXANDRA \\ STANESCU ${ }^{2}$, DANIELA MANUC ${ }^{8}$, CAMELIA C. DIACONU ${ }^{2,9}$
}

\author{
${ }^{1}$ Gastroenterology Clinic, Colentina Clinical Hospital, Bucharest, Romania \\ 2"Carol Davila" University of Medicine and Pharmacy, Bucharest, Romania \\ ${ }^{3}$ Gastroenterology Clinic, University Emergency Hospital Bucharest, Bucharest, Romania \\ ${ }^{4}$ Urology Clinic, Emergency Universitary Central Military Hospital, Bucharest, Romania \\ ${ }^{5}$ Diabetes, Nutrition and Metabolic Diseases, "N.C. Paulescu" Institute, Bucharest, Romania \\ ${ }^{6}$ Surgery Clinic, St. Pantelimon Clinical Emergency Hospital, Bucharest, Romania \\ ${ }^{7}$ Department of Plastic and Reconstructive Microsurgery, Clinical Emergency Hospital of Bucharest, \\ Bucharest, Romania \\ "Carol Davila" University of Medicine and Pharmacy, Faculty of Dental Medicine, Bucharest, Romania \\ ${ }^{9}$ Internal Medicine Clinic, Clinical Emergency Hospital of Bucharest, Bucharest, Romania \\ All authors have equal contributions
}

\begin{abstract}
Paraneoplastic syndromes are disorders induced by the altered immune response to a malignant tumor, which secretes hormones, peptides, or cytokines. The clinical manifestations of the paraneoplastic syndromes are the consequence of the substances produced by the tumor and may affect various organs and systems, being classified in endocrine, neurologic, dermatologic, rheumatologic and hematologic syndromes. These syndromes are most commonly associated with malignancies like small cell lung cancer, breast cancer, gynecologic tumors and hematologic malignancies. Paraneoplastic syndromes may be the first or most obvious manifestation of a neoplasia; if they occur in a patient without a known cancer, they should be carefully investigated. In this regard, they may reveal early a clinically ocult tumor, in a highly treatable stage. The incidence and prevalence of digestive tumors is increasing. Therefore, in order to ensure proper treatment and longer survival, an early diagnosis is necessary. Gastrointestinal cancers are often accompanied by paraneoplastic syndromes, which sometimes may be the "clue" for the diagnosis of cancer. In digestive malignant neoplasms, paraneoplastic dermatologic syndromes are the most common. The evolution of patients with paraneoplastic syndromes depends on patient's comorbidities and complications. If the malignancy is successfully treated, the paraneoplastic syndromes will evolve favorably.
\end{abstract}

Keywords Malignancy, gastrointestinal tumor, dermatologic syndrome, hypercalcemia, hepatocellular carcinoma.

To cite this article: DRAGHICI T, NEGREANU L, BRATU OG, STOIAN AP, SOCEA B, NEAGU TP, STANESCU AMA, MANUC D, DIACONU CC. Paraneoplastic syndromes in digestive tumors: a review. Rom Biotechnol Lett. 2019; 24(5): 813-819. DOI: $10.25083 / \mathrm{rbl} / 24.5 / 813.819$ 


\section{Introduction}

Paraneoplastic syndromes are disorders induced by the altered immune response to a malignant tumor, which secretes hormones, peptides, or cytokines. The clinical manifestations of the paraneoplastic syndromes are the consequence of the substances produced by the tumor and may affect various organs and systems, being classified in endocrine, neurologic, dermatologic, rheumatologic and hematologic syndromes. These syndromes are most frequently associated with malignancies like small cell lung cancer, breast cancer, gynecologic tumors and hematologic malignancies [1-9]. Paraneoplastic syndromes may be the first or most obvious manifestation of a neoplasia; if they occur in a patient without a known cancer, the patient should be carefully investigated. In this regard, they may reveal early a clinically ocult tumor, in a highly treatable stage. Due to the wide spectrum of manifestations, these syndromes have to be managed by a multidisciplinary team, composed of oncologists, surgeons, radiation oncologists, endocrinologists, hematologists, neurologists and dermatologists [10].

Paraneoplastic syndromes are an important cause of morbidity in neoplastic patients. Therefore, prompt treatment can highly improve patients' quality of life and may prolong survival. The treatment mainly adresses the malignancy but also the imunosupression, hydroelectrolytic and hormonal imbalances which occur in endocrine paraneoplastic syndromes.

Decades ago, Hellen Curth proposed some criteria for the definition of the paraneoplastic syndromes, known as the Curth's postulates. These criteria are: the malignant disease and the paraneoplastic syndrome must appear at the same time and have the same clinical evolution, paraneoplastic syndromes should be uncommon relative to the prevalence of the cancer, the association of the paraneoplastic syndrome with the cancer has been previously demonstrated, and the remote cutaneous manifestations are specific to a certain tumor (in the case of a dermatological paraneoplastic syndrome) [11].

It is well known that the incidence and prevalence of digestive tumors is increasing. Therefore, in order to ensure proper treatment and better quality of patients' life, an early diagnosis is necessary. Gastrointestinal cancers are often accompanied by paraneoplastic syndromes, which sometimes may be the „clue” for the diagnosis of cancer.

\section{Types of Paraneoplastic Syndromes}

The paraneoplastic syndromes are classified in: endocrine, neurologic, dermatologic, rheumatologic, and hematologic syndromes. Other types of paraneoplastic syndromes, more rare, are renal, gastrointestinal and cardiovascular syndromes [12].

Paraneoplastic endocrine syndromes: they occur from tumors' aberrant production of hormones, hormone precursors or hormone-like substances and cause metabolic disorders. They are usually diagnosed after the cancer has been detected and they don't correlate with cancer stage. The most common paraneoplastic endocrine syndromes include: SIADH (Syndrome of Inappropriate Antidiuretic Hormone Secretion), hypercalcemia, Cushing syndrome and hypoglycemia [13].

Paraneoplastic neurologic syndromes: the pathogenesis of these syndromes is unknown, the causes might be multifactorial, resulting from immune cross-reactivity between the tumor cells and the nervous system. As a response to malignancy, autoantibodies in the serum and cerebrospinal fluid appear, which are called onconeural antibodies. Also, various substances that affect the nervous system may develop. Unlike paraneoplastic endocrine syndromes, neurologic paraneoplastic symptoms and signs are detected in $80 \%$ of cases before malignancy is diagnosed [14]. Neurologic paraneoplastic syndromes are quite rare, in less than $1 \%$ of neoplastic patients, having a higher incidence in small cell lung cancer, lymphoma or myeloma [14]. Paraneoplastic neurologic syndromes can affect the central nervous system, the neuromuscular junction or the peripheral nervous system. Depending on the affected part, symptoms may include: cognitive and personality changes, ataxia, cranial nervous deficits, weakness or numbness. In neoplastic patients, neurologic manifestations can also occur as a consequence of brain metastases, leptomeningeal disease, spinal cord, nervous root compression and treatment side effects. The most common paraneoplastic neurological syndromes are: limbic encephalitis, paraneoplastic cerebellar degeneration, Lambert-Eaton myasthenia syndrome, myasthenia gravis, autonomic neuropathy and subacute sensory neuropathy. These conditions may also appear in the absence of a malignancy, therefore infectious, toxic or metabolic causes should be excluded [14-15].

Paraneoplastic dermatologic and rheumatologic syndromes: the most frequent are acanthosis nigricans, dermatomyositis, erythroderma, hypertrophic osteoarthropathy, leukocytoclastic vasculitis, paraneoplastic pemphigus, polymyalgia rheumatica, Sweet syndrome, scleroderma, itching, reactivation of latent varicella-zoster virus, adipose nodular necrosis of subcutaneous tissue, and dermic melanosis [16].

Paraneoplastic hematologic syndromes: these syndromes are usually asymptomatic for long time and appear when the disease is already in an advanced stage. They can improve with the proper treatment of the malignancy. The most common paraneoplastic hematologic syndromes are: eosinophilia, granulocytosis, pure red cell aplasia and trombocytosis. Anemia is also very common in malignancies and it is caused by tumors' chronic bleeding, poor absorption of the vitamins and increased destruction or insufficient production of red blood cells [17].

\section{Epidemiology and Etiology}

The causes of paraneoplastic syndromes are not clearly understood. The frequency of these syndromes ranges from $10-15 \%$ to $2-20 \%$ of the malignancies [18]. 
Neurologic paraneoplastic syndromes appear in less than $1 \%$ of neoplastic patients ${ }^{18}$. The predilection of these syndromes for the gender or race is not known. Also, the risk of death and complications in patients with paraneoplastic syndromes is not reported. Paraneoplastic endocrine syndromes are usually diagnosed after the cancer has been detected, without correlation with cancer stage. Neurologic paraneoplastic symptoms and signs are detected in $80 \%$ of cases before malignancy is diagnosed ${ }^{18}$. Renal cell carcinoma and prostate cancer are the most common urological neoplasias associated with paraneoplastic syndromes. They usually appear in the final stage of the cancer and in aggressive tumors, having an unfavorable prognosis. Over $70 \%$ of prostate cancers are associated with endocrine, dermatologic and neurologic paraneoplastic syndromes [19].

In digestive malignant neoplasms, paraneoplastic dermatologic syndromes are the most common. These include: acanthosis nigricans, seborrheic dermatosis, pruritus, pityriasis rotunda, paraneoplastic acrokeratosis of Bazex syndrome, hypertrichosis lanuginosa, dermatomyositis, paraneoplastic pemphigus. Paraneoplastic endocrine syndromes (hypercalcemia, hypoglycemia, thyrotoxicosis), paraneoplastic hematologic syndromes (polycythemia, thrombophlebitis, anemia, porphyria) and paraneoplastic neurologic syndromes (Lambert-Eaton myasthenic syndrome, neuropathy) can also appear in gastrointestinal tumors [20] (Table 1).

Table 1. Paraneoplastic syndromes in digestive malignancies

\begin{tabular}{|c|c|c|c|}
\hline \multicolumn{4}{|c|}{ PARANEOPLASTIC SYNDROMES IN DIGESTIVE MALIGNANCIES } \\
\hline Dermatologic & Endocrine & Hematologic & Neurologic \\
\hline$\checkmark$ Acanthosis nigricans & $\checkmark$ Hypercalcemia & $\checkmark \quad$ Polycythemia & $\checkmark$ Lambert- \\
\hline$\checkmark$ Seborrheic dermatosis & $\checkmark$ Hypoglycemia & $\checkmark$ Thrombophlebitis & Eaton \\
\hline$\checkmark$ Pruritus & $\checkmark$ Tyrotoxicosis & $\checkmark$ Anemia & myasthenic \\
\hline$\checkmark$ Pityriasis rotunda & & $\checkmark$ Porphyria & syndrome \\
\hline$\checkmark$ Acrokeratosis of Bazex & & & $\checkmark$ Neuropathy \\
\hline$\checkmark$ Hypertrichosislanuginosa & & & \\
\hline$\checkmark$ Dermatomyositis & & & \\
\hline$\checkmark$ Pemphigus & & & \\
\hline
\end{tabular}

\section{Esophageal Cancer}

The incidence of small-cell carcinoma of the esophagus ranges between $0.8 \%$ and $2.4 \%$ of all esophageal neoplasms [21]. Small-cell carcinoma is a rare disease, with agressive course and high incidence of metastasis. Casas et al evaluated 199 cases of patients with small-cell carcinoma and have found that the median survival time was 8 months for the patients with limited disease and around 3 months for the patients with extensive disease [21]. As other digestive tumors, esophageal small-cell carcinoma often associates with paraneoplastic syndromes. In 2005, Shimoda et al described the case of a patient with small-cell carcinoma of the esophagus associated with paraneoplastic neurologic syndrome [21]. The neurologic signs and symptomes of sensori-motor neuropathy began 3 years before the malignancy was diagnosed [21].

Esophageal carcinoma is rarely associated with paraneoplastic vasculitis. An article published in 1999 reported the case of a 69 -year-old man who presented fever and numbness of the lower limbs. Symptoms appeared three months before esophageal and gastric carcinomas were diagnosed. After esophagectomy, the fever and numbness disappeared, which suggested a paraneoplastic origin. The histopathologic result confirmed the diagnosis of vasculitis [22].

\section{Gastric Cancer}

Patients with early gastric cancer are asymptomatic in more than $80 \%$ of cases [23]. Most of the patients with advanced cancer present symptoms like weight loss $(60 \%$ of patients) and epigastric pain (50\% of patients) [23]. Other nonspecific symptoms and signs include nausea, vomiting, early satiety, bloating, anorexia, dysphagia and melena. Gastric obstruction is rarely an initial manifestation of the gastric cancer; it can occur in tumors of the antrum and pylorus. Physical examination is usually normal or can point out an epigastric mass, ascites, lymphadenopathy, hepatomegaly, peripheral edema or signs of gastrointestinal hemorrhage. The most common findings are cachexia and signs of bowel obstruction. Laboratories changes are usually identified when the disease is in advanced stages (anemia, a positive test result for fecal occult blood, hypoproteinemia, elevated liver enzymes values). Gastric cancer is occasionally associated with paraneoplastic syndromes. Some of the most common are thrombophlebitis (Trousseau's sign), neuropathies, microangiopathic hemolytic anemia, membranous glomerulonephritis, nephrotic syndrome and disseminated intravascular coagulation. Dermatologic paraneoplastic syndromes, such as hyperpigmented patches in the axilla (acanthosis nigricans), sudden eruption of seborrheic dermatosis (sign of Leser-Trélat), and dermatomyositis may be present $[24,25]$.

The most common sites of metastasis of the gastric cancer are the liver and the peritoneum, but also kidney, bladder, brain, bone, heart, thyroid, adrenal glands and skin [22]. The presence of Virchow's node suggests gastric cancer's spread to the left supraclavicular lymph node, while Sister Mary Joseph's nodule (a periumbilical nodule) indicates metastasis in the periumbilical area. Other sites of 
spread include the pouch of Douglas (Blumer shelf) and the ovaries (Krukenberg's tumor). Unusual signs of metastatic disease are the shoulder-hand syndrome (bone metastasis), diplopia and blindness (orbit and retinal metastases), and virilization (Krukenberg's tumors) [23, 24].

\section{Hepatocellular carcinoma}

Hepatocellular carcinoma (HCC) should be suspected in patients with stable cirrhosis, who decompensate rapidly, with ascites, encephalopathy, jaundice or variceal bleeding. Refractory ascites or hemorrhage might appear with the invasion of the portal vein or, rarely, the hepatic veins, causing severe portal hypertension. HCC must be suspected in all new cases of portal vein thrombosis. Right quadrant upper pain, anorexia with weight loss, vomiting and jaundice are common symptoms found in $\mathrm{HCC}$. Budd-Chiari syndrome (obstruction of the hepatic veins) might also appear in HCC (by tumor invasion) and it is characterized by tender hepatomegaly, tense ascites and abdominal pain. Physical examination and laboratory findings are also important in $\mathrm{HCC}$ - an abdominal mass may be palpable, a bruit related to arterioportal shunting can be heard and alpha-fetoprotein(AFP) levels might be increased [26,27].

Some of the manifestations of HCC are not due to the effects of the tumor or metastases, but to paraneoplastic syndromes. These syndromes are rare and most of them include biochemical abnormalities without associated clinical consequences. Most common paraneoplastic syndromes include hypoglycemia, erythrocytosis and hypercalcemia. One of the most important paraneoplastic syndrome, met in less than $5 \%$ of patients with $\mathrm{HCC}$, is type B hypoglycemia [27]. This syndrome appears in the early stages of the disease and is characterized by severe hypoglycemia. On the other hand, type A hypoglycemia is a less severe form of glycopenia, found in rapidly growing hepatocellular carcinoma. Type A hypoglycemia is also found in other malignant tumors of the liver. Erythrocytosis or polycythemia are another important paraneoplastic syndromes, caused by the synthesis of erythropoietin or erythropoietinlike substance by malignant hepatocytes. It might occur in $3-12 \%$ of patients with hepatocellular carcinoma [27, 28]. Hypercalcemia might also appear as a paraneoplastic syndrome, in the absence of osteolytic metastases, and may be due to the secretion of parathyroid hormone-related protein (PTHrP) by the tumor. If hypercalcemia is severe, complications like drowsiness and lethargy can appear. Another paraneoplastic syndrome, hypercholesterolemia, appears in $10-40 \%$ of patients with hepatocellular carcinoma [28]. Some patients also have thrombocytopenia or leukopenia as paraneoplastic syndromes and not caused by malignant infiltration of bone marrow. Dermatologic paraneoplastic syndromes are rare in HCC. Pityriasis rotunda (circumscripta) refers to single or multiple round or oval, hyperpigmented lesions, on the abdomen, appearing mostly in black Africans. Other paraneoplastic syndromes in patients with HCC or other liver tumors are: dysfibrinogenemia, carcinoid syndrome, neuropathy, osteoporosis, polymyositis, porphyria cutanea tarda, sexual changes (gynecomastia, testicular atrophy and precocious puberty), systemic arterial hypertension, thyrotoxicosis, thrombophlebitis migrans and watery diarrhea syndrome $[28,29]$.

\section{Tumors of the Small Intestine}

Most neoplasms of the small intestine are asymptomatic, being diagnosed incidentally or late in their evolution. Patients with adenocarcinoma of the duodenum usually present frank or occult gastrointestinal hemorrhage, abdominal pain and biliary obstruction. They will rarely manifest symptoms of intestinal obstruction. Instead, patients with adenocarcinoma of the jejunum and ileum have mild complaints, such as abdominal pain, nausea, vomiting, bloating and occult bleeding. Adenomas, adenocarcinomas and lymphomas might cause volvulus and obstruction by growing into the lumen. Chronic gastrointestinal bleeding is the second most common symptom of small intestine neoplasms, massive hemorrhage being commonly met in sarcomas (GIST). $25 \%$ of the patients with small intestinal malignancy have a palpable abdominal mass at physical examination and signs of obstruction: distention, borborygmi and mild to moderate abdominal tenderness [30]. There are not paraneoplastic syndromes described in small intestinal malignancies.

\section{Colorectal Cancer}

Colorectal cancer is the third most common malignancy in the world [30]. Risk factors include age (more than 50 years old, physical inactivity, obesity, diet with increased red meat and decreased fiber, history of polyps or colorectal cancer, inflammatory bowel disease, hereditary syndromes (familial adenomatous polyposis and hereditary nonpolyposis colorectal cancer). The only paraneoplastic syndromes described in colorectal cancer are the dermatologic syndromes. Gastrointestinal bleeding, abdominal pain, change in bowel habits, obstruction, anorexia with weight loss are the most frequent symptoms and signs. Any iron deficiency should be evaluated with upper and lower endoscopy [31].

\section{Pancreatic Cancer}

Pancreatic endocrine tumors may lead to Cushing's syndrome. The syndrome may appear in gastrinomas, when it is a negative prognostic factor. In pancreatic endocrine tumors, hypercalcemia may appear as a paraneopastic syndrome, due to the release of parathyroid hormonerelated peptide (PTHrP). These tumors are usually large and liver metastases might also appear. Pancreatic endocrine tumors secreting calcitonin hormone have been described. Patients have diarrhea that disappears with the resection of the tumor [30]. Thrombophlebitis as a paraneoplastic syndrome may appear in pancreatic cancer [31]. 


\section{Cutaneous Manifestations of Gastro- intestinal Malignancies}

Paraneoplastic pemphigus, acanthosis palmari, acanthosis nigricans, paraneoplastic acrokeratosis of Bazex and hypertrichosis lanuginosa are some of the most frequent paraneoplastic dermatologic syndromes in digestive malignancies.

Paraneoplastic pemphigus appears in multiple neoplasms, such as gastrointestinal malignancies, lymphomas and leukemias, thymomas and soft tissue sarcomas. It is characterized by painful mucosal erosions, skin eruption, intraepidermal acantholysis, keratinocyte necrosis, deposition of IgG, serum autoantibodies and immunoprecipitation of a complex of four proteins from keratinocytes. The prognosis of paraneoplastic pemphigus depends on the type of associated malignancy.

Acanthosis palmaris, pachydermatoglyphy, palmar hyperkeratosis or palmar keratoderma represent a paraneoplastic syndrome which manifests by velvety texture with dermatoglyphics or by a cobbled surface of the palms and fingers. $91 \%$ of the cases appear in association with a malignancy [16]. These dermatologic paraneoplastic phenomenons are usually met in gastric or lung cancers (more than $25 \%$ of all the malignancies) $[16,17]$.

Acanthosis nigricans is characterized by hyperplasia and hyperpigmentation of the skin of the neck and axillae. It may be associated with skin tags. When it is associated with an internal malignancy, the cutaneous extent may be severe, affecting also the hands, genital organs and the oral mucosa. Abdominal adenocarcinomas represent more than $85 \%$ of the associated malignancies, while gastric carcinomas appear in more than $60 \%$ of the cases [11].

Paraneoplastic acrokeratosis of Bazex is a paraneoplastic syndrome that associates with a neoplasm of the upper aerodigestive tract. It manifests with skin eruption (including the tip of the nose and ears; in an advanced stage of the disease, face and scalp are affected) and marked nails dystrophy. From 50 cases of patients with acrokeratosis of Bazex, all of them have had a malignancy, including esophageal carcinoma or gastric carcinoma with cervical metastases [11].

Hypertrichosis lanuginosa is a rare paraneoplastic syndrome characterized by thin, unpigmented lanugo-type hair on the face, forehead, ears, nose, axillae, limbs and trunk and by glossodynia, papillary hypertrophy of the tongue, disturbances of taste and smell, diarrhea, scleroderma, acanthosis nigricans, seborrheic keratoses, adenopathy and weight loss. Lung cancer and colorectal carcinomas are frequently associated with this paraneoplastic syndrome $[10,16]$.

\section{Autoantibodies in Paraneoplastic Syndromes}

Pathophysiology of paraneoplastic syndromes is very complex and disputed. When a tumor is identified by the organism, antibodies are produced against the tumor cells.
Those antibodies may cross-react with normal tissues and destroy them, too. This reaction is called a paraneoplastic disorder [31]. Paraneoplastic syndromes may also appear when the tumor itself produces and releases active substances like hormones, hormone precursors, enzymes or cytokines. There are multiple neoplasms that produce proteins which are expressed in utero by embryonic and fetal cells, but not by the normal adult cells - these proteins are very useful as tumor markers (carcinoembryonic antigen [CEA], alpha-fetoprotein [AFP], carbohydrate antigen 19-9 [CA 19-9]) [32].

Some examples of autoantibodies found in paraneoplastic syndromes are: Anti-Hu (autoantibody identified in the serum of the patients with paraneoplastic sensory neuropathy or encephalomyelitis; Anti-Ri (in patients with opsoclonus/myoclonus syndrome); antineuronal antibodies Ma1 and Ma2 (brain-specific or testis-specific proteins); anti-Yo or anti-Purkinje cell antibody 1 (in paraneoplastic cerebellar degeneration).

\section{Treatment of Paraneoplastic Syndromes}

The treatment of paraneoplastic syndromes depends on their location and type. There are two therapeutic strategies. The first one is the management of the malignant tumor responsible for the paraneoplastic syndrome. The same protocols are applied as in patients without any paraneoplastic syndromes, such as surgery, radiation or chemotherapy. Surgery in patients with paraneoplastic syndromes is adressed to the underlying neoplasm. There are cases when paraneoplastic disorders may resolve without tumor resection. On the other hand, in patients with ectopic adrenocorticotropic hormone (ACTH) syndrome, bilateral adrenalectomy with hormone replacement is the most useful treatment [33]. Also, in patients with thymoma, complete resection of the tumor must be performed in order to obtain the paraneoplastic syndrome remission. Monitoring and perioperative care, before and after radiotherapy or chemotherapy, are definitely needed [2, 29].

The second therapeutic strategy, applied in patients with confirmed serum antibodies, is represented by immunosuppresion, using intravenous immunoglobulins, steroids, other immunosuppressive drugs or plasma exchange. If autoantibodies are found, the best drug to use is cyclosporine. It was demonstrated that some patients with paraneoplastic syndromes have a positive response when using rituximab, but further studies are required [28]. Other possibilities of treatment for paraneoplastic syndromes are lymphocyte immune globulins, which suppress the immune reactions, or corticosteroids (prednisone), which decrease inflammation.

\section{Prognosis of Paraneoplastic Syndromes}

The evolution of patients with paraneoplastic syndromes depends on patient's comorbidities and on complications that may occur during the disease evolution 
[32, 34, 35]. If the malignancy is successfully treated, paraneoplastic syndromes will evolve favorably. Prognosis of paraneoplastic syndromes may vary greatly, because paraneoplastic syndromes differ from individual to individual. In a review of patients with paraneoplastic pemphigus, infection was often a major cause of death $[18,36]$. Also, disseminated intravascular coagulation indicates a poor prognosis, while hypertrophic osteoarthropathy is one of the few paraneoplastic syndromes with a favorable prognosis [18, 37, 38]. Also, some paraneoplastic syndromes can spontaneously cure. Underlying malignancy or irreversible organ damage, in the end, may lead to death, as in patients with acute heart failure or kidney failure.

\section{Conclusions}

Paraneoplastic syndromes, a very intriguing and complex subject, affect the clinical condition and the response to treatment of patients with neoplasms. The incidence of these syndromes will probably increase, as the prognosis of cancer patients will be better, with a longer survival. Early recognition and diagnosis of paraneoplastic syndromes in digestive malignancies is very important, in order to improve patients' prognosis and quality of life.

\section{Acknowledgements: none}

\section{References}

1. S.H. GULTEKIN. Recent developments in paraneoplastic disorders of the nervous system. Surg Pathol Clin 8(1):89-99 (2015).

2. B. PARASCHIV, C.L. TOMA, C. DIACONU. Bronchiolo-alveolar carcinoma in a young patient: a case report. Arch Bronconeumol 49(7):315-316 (2013).

3. B. PARASCHIV, C. DIACONU, C. TOMA, M. BOGDAN. Paraneoplastic syndromes: the way to an early diagnosis of lung cancer. Pneumologia 64(2):14-19 (2015).

4. C.C. DIACONU, D. ARSENE, A. BĂLĂCEANU, D. BARTOŞ. A rare tumor revealed by abdominal trauma: case presentation. Rom J Morphol Embryol 55(3):973-976 (2014).

5. A. BĂLĂCEANU, C. DIACONU, G. ARON. Budd-Chiari syndrome as initial presentation of hepatocellular carcinoma - value of ultrasound. Med Ultrason 16(2):172-174 (2014).

6. A. BĂLĂCEANU, C. DIACONU, D. MATEESCU, A. STĂNICĂ. Hepatocellular carcinoma with hepatic and pulmonary metastasis, inferior vena cava and left pulmonary artery thrombosis in a patient with asymptomatic hepatitis C. Case report. Med Ultrason 12(4): 345-348 (2010)

7. C.C. DIACONU, D. ARSENE, B. PARASCHIV, A. BĂLĂCEANU, D. BARTOŞ. Hyponatremic encephalopathy as the initial sign of neuroendocrine small cell carcinoma - case report. Acta Endo IX(4): 637-642 (2013).

8. C. DIACONU, A. BĂLĂCEANU. Hyponatremia in cancer patients: much more than a common electrolytic disorder. Arch Balk Med Union 51(1):50-54 (2016).

9. I.C. DUMITRAŞ, C. IONESCU, D. BARTOŞ, C. DIACONU. The diagnosis of malignant disease: sometimes a matter of pure chance. Arch Balk Med Union 52(1):112-116 (2017).

10. B.T. BILYNSKY, M.B. DZHUS, R.I. LITVINYAK. The conceptual and clinical problems of paraneoplastic syndrome in oncology and internal medicine. Exp Oncol 37(2):82-8 (2015).

11. H.O. CURTH, A.W. HILBERG, G.F. MACHACEK. The site and histology of the cancer associated with malignant acanthosis nigricans. Cancer 15:364-382 (1962)

12. L.W. BAIJENS, J.J. MANNI. Paraneoplastic syndromes in patients with primary malignancies of the head and neck: four cases and a review of the literature. Eur Arch Otorhinolaryngol 263: 32-36 (2006)

13. S.H. RALSTON, S.J. GALLACHER, U. PATEL, J. CAMPBELL, I.T. BOYLE. Cancer-associated hypercalcemia: morbidity and mortality. Clinical experience in 126 treated patients. Ann Intern Med 112: 499-504 (1990).

14. A. DIDELOT, J. HONNORAT. Update on paraneoplastic neurological syndromes. Curr Opin Oncol 21: 566-572 (2009).

15. D.P. RICHMAN, M.A. AGIUS. Treatment of autoimmune myasthenia gravis. Neurology 61:1652-1661 (2003).

16. B.H. THIERS, R.E. SAHN, J.P. CALLEN. Cutaneous manifestations of internal malignancy. CA Cancer $J$ Clin 59: 73-98 (2009).

17. E. SIERKO, M.Z. WOJTUKIEWICZ. Platelets and angiogenesis in malignancy. Semin Thromb Hemost 30:95-108 (2004).

18. S. LEGER, D. PICARD, S. INGEN-HOUSZ-ORO, J.P. ARNAULT, F. AUBIN, F. CARSUZAA, G. CHAUMENTIN, J. CHEVRANT-BRETON, O. CHOSIDOW, B. CRICKX. Prognostic factors of paraneoplastic pemphigus. Arch Dermatol 148(10):1165-72 (2012).

19. M.K. HONG, J. KONG, B. NAMDARIAN, A. LONGANO, J. GRUMMET, C.M. HOVENS, A.J. COSTELLO, N.M. CORCORAN. Paraneoplastic syndromes in prostate cancer. Nature Reviews Urology 7(12): 681-692 (2010).

20. M. FELDMAN, L.S. FRIEDMAN, L.J. BRANDT, W.O. TSCHUMY JR. Oral disease and oral-cutaneous manifestations of gastrointestinal and liver disease. Sleisenger and Fordtran's Gastrointestinal and liver disease 22:358-365.(2010).

21. K.L. BEYER, J.B. MARSHALL, A.A. DIAZ-ARIAS, T.S. LOY. Primary small cell carcinoma of the esophagus. J Clin Gastroenterol 13:135-41 (1991). 
22. T. MITA, Y. NAKANISHI, A. OCHIAI, T. SHIMODA, H. KATO, H. YAMAGUCHI, G. TODA. Paraneoplastic vasculitis associated with esophageal carcinoma. Pathology International 49(7): 643-647 (1999).

23. T. YAMADA, J.M. INADOMI, R. BHATTACHARYA, J.A. DOMINITZ, J.H. HWANG. Tumors of the stomach. Yamada's Handbook of Gastroenterology third edition 23:255-256 (2013).

24. M. FELDMAN, L.S. FRIEDMAN, L.J. BRANDT, W.O. TSCHUMY JR. Adenocarcinoma and other tumors of the stomach. Sleisenger and Fordtran's Gastrointestinal and liver disease 54:898-890 (2010).

25. C. DIACONU, A. SOPA, A. BĂLĂCEANU. Dermatoze paraneoplazice în neoplaziile digestive. Medicina Modernă XIII(4):170-174 (2006).

26. M. FELDMAN, L.S. FRIEDMAN, L.J. BRANDT, W.O. TSCHUMY JR. Tumors and cysts of the liver. Sleisenger and Fordtran's Gastrointestinal and liver disease 94: 1571-1573 (2010).

27. T. YAMADA, J.M. INADOMI, R. BHATTACHARYA, J.A. DOMINITZ, J.H. HWANG. Hepatocellular carcinoma. Yamada's Handbook of Gastroenterology third edition 43: 444-446 (2013).

28. D.L. KASPER, S.L. HAUSER, J.L. JAMESON, A.S. FAUCI, D.L. LONGO, J. LOSCALZO. Tumors of the liver and biliary tree. 19th Edition Harrison's principles of internal medicine 111: 544-548 (2015).

29. H. GODARA, A. HIRBE, M. NASSIF, H. OTEPKA, A. ROSENSTOCK. Liver diseases. The Washington Manual of Medical Therapeutics 19: 909-910 (2014).

30. A. BĂLĂCEANU, C. DIACON, G. ARON. BuddChiari syndrome as initial presentation of hepatocellular carcinoma - value of ultrasound. Med Ultrason 16(2):172-174 (2014).

31. C. DIACONU, D. MATEESCU, A. BĂLĂCEANU, M. MARCU, V. JIANU, A. STĂNICĂ. Pancreatic cancer presenting with paraneoplastic thrombophlebitis: a case report. J Med Life 3(1): 96-99 (2010).
32. A.N. CIUHU, R.A. RAHNEA NIȚĂ, M. POPESCU, C.M. BADIU, A.M. PANTEA STOIAN, D. LUPULIASA, F. GHERGHICEANU, C. DIACONU, G. RAHNEA-NITA. Evidence of strong opioid therapy for palliation of breathlesness in cancer patients. Farmacia 65(2):173-178 (2017).

33. C. POIANA, M.C. NEAMTU, E.T. AVRAMESCU, M. CARSOTE, R. TRIFANESCU, D. TERZEA, O.M. NEAMTU, R. DANCIULESCU MIULESCU. The dedifferentiation of neuroendocrine tumor metastases: myth or reality? Romanian Journal of Morphology and Embryology, 54(1): 201-203 (2013).

34. C. POIANA, M.C. NEAMTU, E.T. AVRAMESCU, M. CARSOTE, R. TRIFANESCU, D. TERZEA, O.M. NEAMTU, D. FERECHIDE, R. DANCIULESCU MIULESCU. The poor prognosis factors in G2 neuroendocrine tumor. Romanian Journal of Morphology and Embryology, 54(3)S:717-720 (2013).

35. O. GINGHINĂ, C. NEGREI, A. HUDIT,Ă, V. IOANALAVRIC, B. GĂLĂȚEANU, S. DRAGOMIR, G.T.A. BURCEA DRAGOMIROIU, M. BÂRCĂ, C. NIȚIPIR, C.C. DIACONU, A.M. PANTEA STOIAN, N. IORDACHE, A. BĂLĂNESCU. In vitro impact of some natural compounds on HT-29 colorectal adenocarcinoma cells. Farmacia, 65(6): 947-953 (2017).

36. C. DIACONU, A. BĂLĂCEANU, E. MOROŞAN. Sepsis biomarkers: past, present and future. Farmacia, 63(6):811-815 (2015).

37. C.P. TĂTARU, R. IANCU, A. DOGĂROIU, C. DIACONU, C. CORBU. Evaluation of the efficacy and safety of intravitreal bevacizumab for macular edema related to retinal vein occlusion. Farmacia, 64(3):444-448 (2016).

38. NITIPIR C, ANA NEAGU M, IACIU C, BARBU MA, POPESCU BC, ORLOV C, POPA AM, CONSTANTINESCU R, PIETROSANU C, PITURU S, HAINAROSIE R, STOIAN AMP, STANCIU AE. New biomolecules for the treatment of Disseminated Intravascular Coagulation. Rom Biotechnol Lett 24(4): 580-585 (2019). 